\title{
Aberrant positioning of a central venous dialysis catheter to reveal a left-sided partial anomalous pulmonary venous connection
}

\author{
Manohar R Chintu \\ Shammikumar Chinnappa \\ Sunil Bhandari \\ Department of Nephrology, Hull and \\ East Yorkshire Hospitals NHS Trust, \\ Kingston upon Hull, United Kingdom
}

\begin{abstract}
We describe the identification of a rare, left-sided, partial anomalous pulmonary venous connection during routine central venous catheterization. To our knowledge, this is the first report in the literature to describe this anomaly in a hemodialysis patient. A young man had anomalous connection of the veins draining the upper lobe of the left lung and left innominate vein. Our case demonstrates the importance of routine fluoroscopy during insertion of central venous catheters to detect these anomalies and minimize complications.
\end{abstract}

Keywords: hemodialysis, central venous catheters, fluoroscopy, anomaly, veins

\section{Case report}

A 33-year-old man with known end stage renal failure requiring hemodialysis attended the vascular access clinic for elective insertion of a semi-permanent central venous dialysis catheter (permacath). The catheter was inserted into the left internal jugular vein under ultrasound guidance (Site-Rite ${ }^{\circledR} 4$ ultrasound system, USA) with no apparent difficulty. Good blood flows were demonstrated in both catheter lumens and there was no difficulty extracting blood from either one. A subsequent check chest X-ray performed immediately after the procedure revealed an abnormal anatomical position of the central catheter. Instead of passing into the innominate vein, the catheter appeared radiologically located in the left hemi thorax (Figure 1). Venography confirmed an anomalous pulmonary venous connection between the left innominate vein and the left pulmonary vein draining the upper lobe of the lung (Figure 2). This catheter was removed and a further dialysis central catheter was inserted under fluoroscopic guidance without complication. The patient remained asymptomatic and hemo-dynamically stable throughout this period.

\section{Discussion}

Partial anomalous pulmonary venous connection (PAPVC) is a rare congenital abnormality in which one or more (but not all) pulmonary veins drain into the right atrium or its tributaries (superior vena cava, inferior vena cava, left innominate vein) (Senocak et al 1994). PAPVC accounts for about $0.5 \%$ of congenital cardiac defects (Demos et al 2004) commonly associated with atrial septal defects (Senocak et al 1994). This abnormality involves the right lung more often than the left, with a variable ratio of between 2 and 10:1 (Senocak et al 1994; Greene and Miller 1986). In the embryo, anomalous pulmonary venous drainage results from a failure of the connection between the primitive pulmonary splanchnic plexus and the common pulmonary vein derived from the primitive left atrium. Most of these anomalies are usually discovered incidentally during routine chest radiographs or computed tomography (CT) scans of the lungs for other reasons (Demos et al 2004).
Department of Nephrology, Hull and East Yorkshire Hospitals NHS Trust, Kingston upon Hull, United Kingdom, HU32JZ

Tel +44 I482 674566

Fax +44 I482 674998

Email sunil.bhandari@hey.nhs.uk 


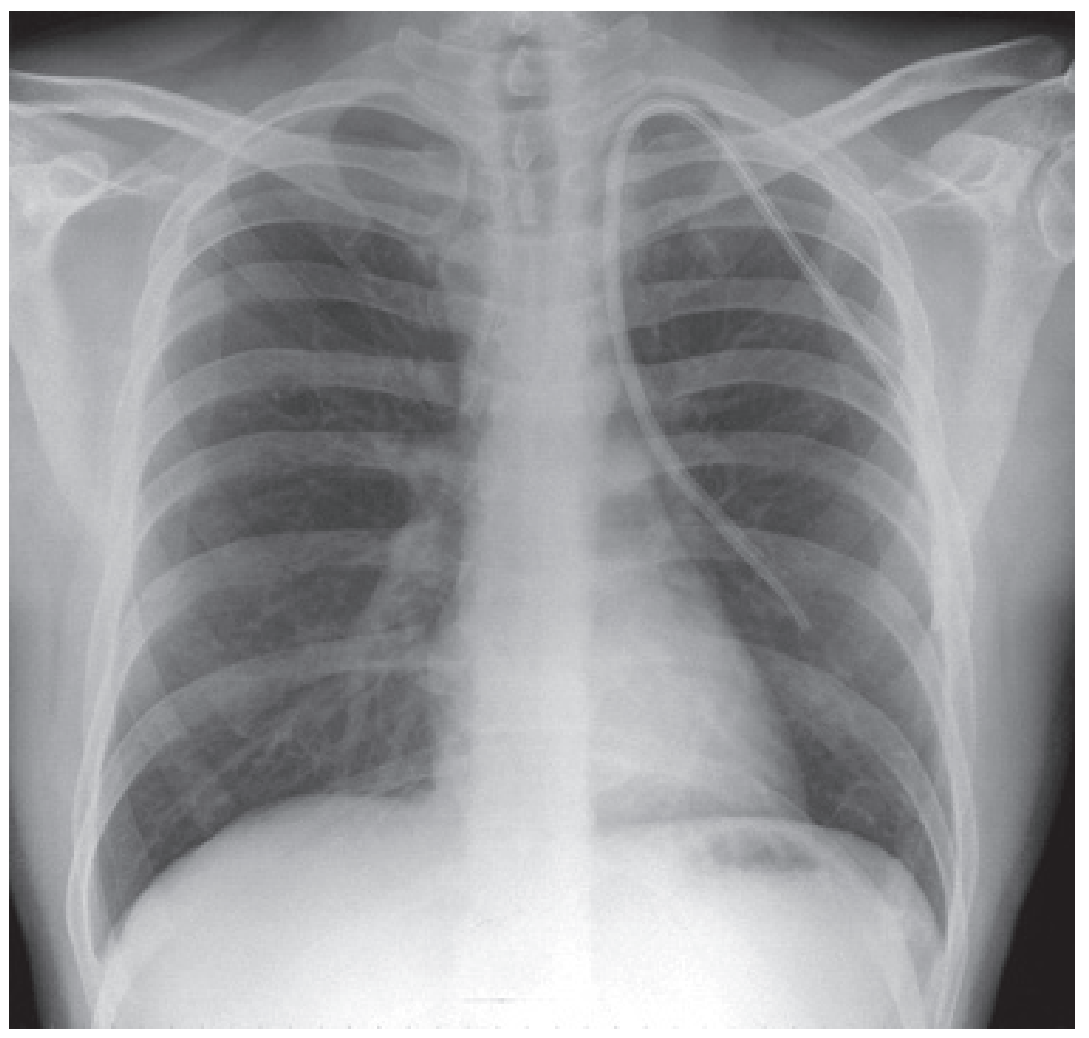

Figure I Chest X-ray depicting abnormal position left internal jugular catheter.

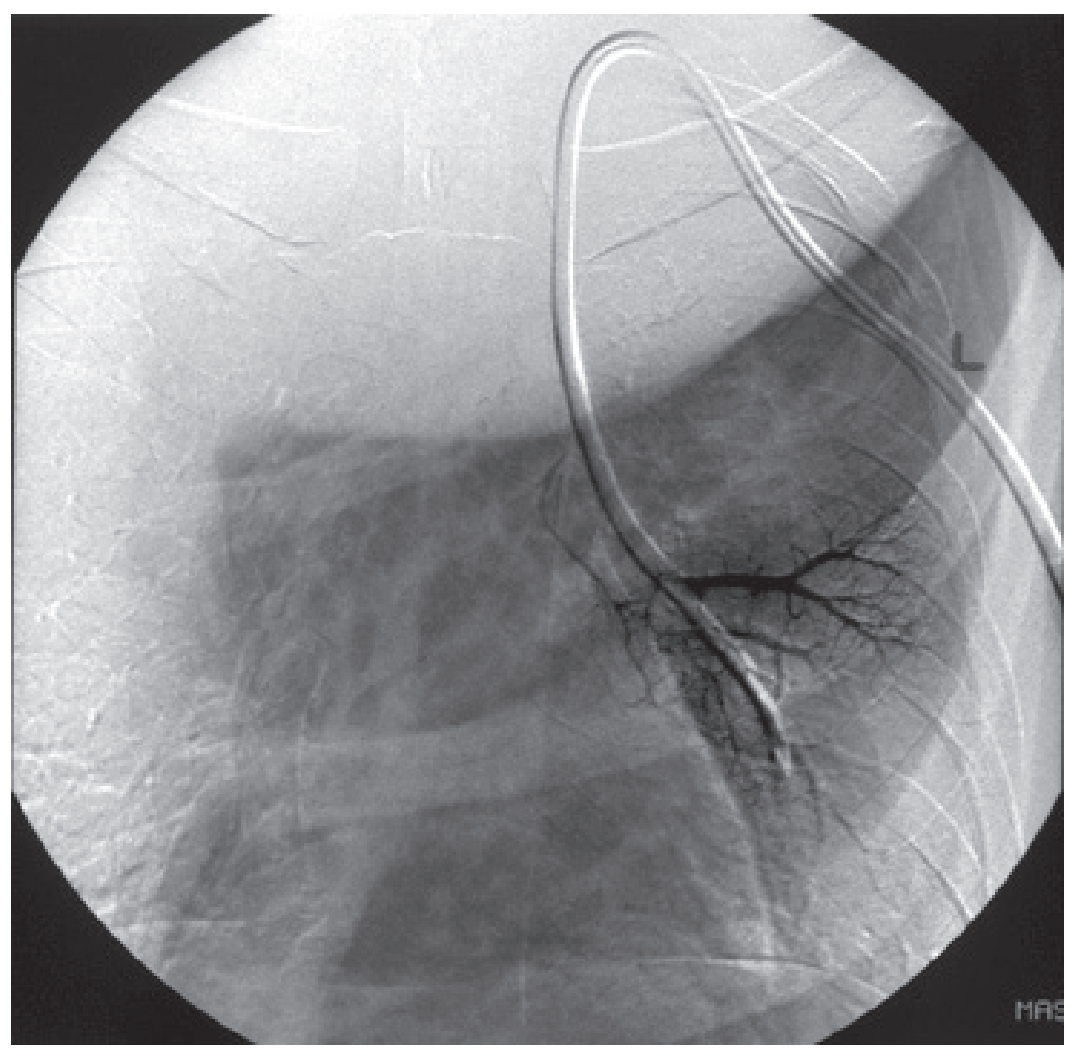

Figure 2 Venogram demonstrating partial anomalous venous return from left upper lobe. 
PAPVC of the left lung is normally asymptomatic and is usually discovered as an incidental finding in adults with normal hearts during routine imaging for other reasons (Demos et al 2004). Hence most anomalies of this kind tend to go undetected. An anomalous venous return on the left side usually drains into a left vertical vein and then returns to the innominate vein (Greene and Miller 1986). This entity has a characteristic appearance on CT (Greene and Miller 1986) and chest radiographs (Alder and Silverman 1973). In an isolated PAPVC, the patient is usually asymptomatic if the anomalous venous return is less than $50 \%$ of total pulmonary venous blood (Greene and Miller 1986). Patients may develop cardio-respiratory symptoms if there is significant left-to-right shunt, which is associated with other cardiac anomalies $(10 \%-15 \%$ of those who have atrial septal defect, also have PAPVC). The majority of patients who have a left PAPVC have a good long-term prognosis. The use of sonosite and chest X-ray checks have minimized the complications associated with central venous line insertions, although they are not $100 \%$ infallible. Despite good blood flows and a normal sonosite, this paper demonstrates the importance of routine fluoroscopy during placement of tunneled catheters, so that such abnormalities can be detected at the time of insertion. Moreover, to function correctly, accurate tip placement of these catheters under fluoroscopic guidance is essential.

\section{Disclosure}

The authors report no conflicts of interest in this work.

\section{References}

Adler SC, Silverman JF. 1973. Anomalous venous drainage of the left upper lobe. A radiographic diagnosis. Radiology, 108:563-5.

Demos TC, Posniak HV, Pierce KL, et al. 2004. Venous anomalies of the thorax. AJR Am J Roentgenol, 182:1139-50.

Greene R, Miller SW. 1986. Cross-sectional imaging of silent pulmonary venous anomalies. Radiology, 159:279-81.

Senocak F, Ozme S, Bilgic A, et al. 1994. Partial anomalous pulmonary venous return. Evaluation of 51 cases. Jpn Heart J, 35:43-50. 
